\title{
The expression and significance of the enhancer of zeste homolog 2 in lung adenocarcinoma
}

\author{
YIJING LV $^{1 *}$, CUNZHONG YUAN ${ }^{2 *}$, XIAOYAN XIAO ${ }^{3}$, XUPING WANG $^{4}$, \\ XUMING JI ${ }^{5}$, HUAYUN YU ${ }^{5}$, ZHICHUN WU ${ }^{5}$ and JIDONG ZHANG ${ }^{1}$ \\ ${ }^{1}$ Department of Traditional Chinese Medicine, ${ }^{2}$ Gynecological Oncology Key Library of Shandong Province, \\ ${ }^{3}$ Department of Nephrology, and ${ }^{4}$ Laboratory of Cardiovascular Remodeling and Function Research, \\ Qilu Hospital, Shandong University, Jinan 250012; ${ }^{5}$ School of Basic Medicine, Shandong \\ University of Traditional Chinese Medicine, Jinan 250014, P.R. China
}

Received February 20, 2012; Accepted April 5, 2012

DOI: $10.3892 /$ or.2012.1787

\begin{abstract}
Lung adenocarcinoma, with increased incidence in the world, exhibits poor prognosis and is usually resistant to conventional chemotherapy due to drug resistance. The enhancer of zeste homologue 2 (EZH2), a histone methyltransferase, plays a key role in tumorigenesis and cancer development through chromatin remodeling in various types of cancer. However, its potential role in lung adenocarcinoma has not been well defined. In this study, the expression of EZH2 was examined in lung adenocarcinoma tissues and cell lines. Most interestingly, EZH2 overexpression was detected in tumor tissue and significantly correlated with histological differentiation, pathological tumor-node-metastasis stage and smoking history, but not with gender or age. Furthermore, EZH2 overexpression was also detected in cisplatin-resistant cancer cells rather than cisplatin-sensitive cells. Short hairpin RNA targeted against EZH2 inhibited cell proliferation and migration, and led to $\mathrm{G}(2) / \mathrm{M}$ cell cycle arrest and apoptosis in both cisplatin-resistant and cisplatin-sensitive cell lines. Moreover, EZH2 knockdown enhanced cisplatin sensitivity of cisplatin-resistant cells and reduced the expression levels of multidrug resistance-related protein 1. Our study suggests that EZH2 contributes to the progression of lung adenocarcinoma, and the deletion of EZH2 inhibits cancer and resensitizes cells to cisplatin in lung adenocarcinoma.
\end{abstract}

Correspondence to: Dr Jidong Zhang, Department of Traditional Chinese Medicine, Qilu Hospital, Shandong University, 107 Wenhua Xi Road, Jinan 250012, Shandong Province, P.R. China

E-mail: jidongzhang1974@163.com

*Contributed equally

Key words: lung adenocarcinoma, enhancer of zeste homolog 2, short hairpin RNA

\section{Introduction}

Lung cancer (LC) is a leading cause of cancer-mortality worldwide. Current medical therapy is relatively effective, but the 5-year survival rate is only $14 \%$ (1). Lung adenocarcinoma (LA) is one major type of LC and accounts for $40 \%$ of nonsmall cell LC (NSCLC) (2). With increased smoking and air pollution, the incidence of LA is increasing in the world (3) including China (4). Moreover, LA usually responds poorly to chemotherapy (5) and most patients of LA are resistant to cisplatin (DDP). Therefore, better defining the pathogenesis of LA and exploring novel therapeutic targets sensitive to chemotherapy are urgently required.

The gene of enhancer of zeste homolog 2 (EZH2), encoding a polycombgroup $(\mathrm{PcG})$ protein, is thought to have the potential to silence genes involved in tumorigenesis. Overexpression of EZH2 has been reported in metastatic prostate cancer and breast cancer, and considered to have diagnostic and prognostic value (6-8). Recent studies indicate that EZH2 is a key component of the polycomb-repressive complex required for maintenance of a stem cell state, and overexpression of EZH2 is involved in drug resistance in ovarian cancers (9). However, the role of EZH2 in cell proliferation, migration, cell cycle regulation and apoptosis remains unclear in LA.

The development of multidrug resistance (MDR) to cancer chemotherapy is a major obstacle to the medical treatment of LA. Multidrug resistance 1 (MDR1), multidrug resistancerelated protein 1 (MRP1) and lung resistance protein (LRP) are thought to be associated with the development of MDR (10-12). However, the relationship between the effect of EZH2 and the development of MDR to LA chemotherapy is still unknown.

In our study, we investigated the EZH2 expression in LA specimens, corresponding distant normal tissues and LA cell lines including cisplatin-resistant cell line (A549/ DDP) and cisplatin-sensitive cell line (A549), and studied its correlation with the clinicopathological features. Furthermore, we investigated the effects of EZH2 depletion on cell proliferation, migration, cycle control and apoptosis regulation in LA cell lines in vitro. We used EZH2 short hairpin RNA (shRNA) to silence EZH2 gene and further 
Table I. Primers used for the RT-PCR.

\begin{tabular}{ll}
\hline Gene & \multicolumn{1}{c}{ Sequence of primer } \\
\hline EZH2 & F: 5'-GCGGGACTAGGGAGTGTTC-3' \\
& R: 5'-AGCAACTGCATTCAGAGTCTT-3' \\
3-actin & F: 5'-GACAGGATGCAGAAGGAGATTACT-3' \\
& R: 5'-TGATCCACATCTGCTGGAAGGT-3' \\
MDR1 & F: 5'-CCCATCATTGCAATAGCAGG-3' \\
& R: 5'-GTTCAAACTTCTGCTCCTGA-3' \\
MRP1 & F: 5'-AGTTCTGCGGTGCTGTTGTG-3 \\
& R: 5'-TTCGCTGAGTTCCTGCGTAC-3' \\
LRP & F: 5'-CATCATTCGCACTGCTGTCT-3' \\
& R: 5'-TTTCTCGGCTTCTGACTGGT-3' \\
\hline
\end{tabular}

analyzed the effects of EZH2 knockdown on acquired cisplatin resistance.

\section{Materials and methods}

Human tissues and clinical data. Neoplastic tissues and distant normal tissues ( $>5 \mathrm{~cm}$ from the margin of the tumor) were obtained from 69 patients (age, range 41-73 years; gender, 36 men and 33 women) who underwent a surgical resection of LA at Qilu Hospital, Shandong University from the year 2004 to 2009. The samples were divided into two halves, one half was fixed in formalin and embedded with paraffin and the other half was stored at $-80^{\circ} \mathrm{C}$ until use. After histological examination, tumor samples were classified as well differentiated $(n=22)$, moderately differentiated $(n=28)$ and poorly differentiated $(n=19)$. The pathological stage (pStage) was made based on American Joint Committee on Cancer guidelines for postoperative tumor-node-metastasis (TNM) classification. Written informed consent was obtained from each patient. This study was approved by the Medical Ethics Committee of the Qilu Hospital, Shandong University.

Immunohistochemistry staining. Specimens were deparaffinized and rehydrated. After antigen retrieval, the primary antibody against EZH2 mAb ab3748 (Abcam, USA) was used at dilution of 1:1000 and incubated overnight at $4^{\circ} \mathrm{C}$. The second antibody from SP reagent kit (Zhongshan Biotechnology Co., Beijing, China) were employed to visualize antibody binding and stained with DAB. The stained slides were reviewed and scored independently by two pathologists who had no knowledge of patient information, and both observers re-examined the immunostained slides with discrepant scores to determine a consensus score. Expression of EZH2 was scored semiquantitatively by the combination of intensity and proportion of the positively stained tumor cells as follows: negative expression, no staining; $1+,<5 \%$ tumor cells staining; $2+, 5-25 \%$ tumor cells staining; $3+, 26-50 \%$ tumor cells staining; and $4+,>50 \%$ tumor cells staining.

Cell culture, EZH2 shRNA transfection, cell migration, and cell proliferation. The study included human lung adeno- carcinoma cell lines A549 and A549/DDP. A549 cell lines (CCL-185) were obtained from the American Type Culture Collection (ATCC, Manassas, VA, USA) and cultured according to the protocol provided by the ATCC. A549/DDP cell line, resistant to cisplatin, was generously provided by the Beijing Cancer Institute (Beijing, China). The A549/DDP clones showed 9-fold greater drug resistance to cisplatin than A549 cells and it was cultured with $6 \mathrm{nM}$ cisplatin (Sigma) to maintain the drug resistance phenotype.

The sequence of EZH2 mRNA from 461-481nt was selected for RNAi target, and the shRNA plasmid for EZH2 gene was constructed according to the protocol of pSuper RNAi System ${ }^{\mathrm{TM}}$ from the OligoEngine Co. A549 cell was transfected with pSuper-shEZH2 vectoror empty pSuper vector as the control using Lipofactamine 2000 transfection reagent (Invitrogen, Carlsbad, CA, USA) according to the manufacturer's instructions. Transfectants were screened with hygromycin B (Roche, Indianapolis, IN, USA).

For proliferation assay, cells were seeded in a 96-well plate with $200 \mu 1$ medium. 3-(4,5)-dimethylthiazol(-2-y1)3,5-diphenyl tetrazolium bromide (MTT, Sigma) was added at concentration of $0.5 \mathrm{mg} / \mathrm{ml}$ and then incubated for $4 \mathrm{~h}$ at $37^{\circ} \mathrm{C}$. The medium was removed, and dimethyl sulfoxide (DMSO, Sigma) was added to each well to dissolve crystals by shaking the plate gently for $30 \mathrm{~min}$ in the dark. The OD value in each well was determined at $570 \mathrm{~nm}$ by a Microplate reader (Thermo Varioskan Flash).

The migration assay was performed using the transwell system (24-wells, $8 \mu \mathrm{m}$ pore size with polycarbonate membrane, BD Biosciences, San Jose, CA, USA) and followed the BD protocol. Cells were stained with DAPI (Beyotime Biotechnology, China). DAPI is known to form fluorescent complexes with natural double-stranded DNA in nuclear, showing a blue fluorescence. The total number of cells invading and adhering to the lower surface was counted in 6 representative fields under an Olympus light microscope.

RNA isolation and reverse transcription PCR. RNA isolation and RT-PCR was performed as previously described (13). Total RNA was extracted from LA cell lines using TRIzol reagent (Invitrogen) based on the suggested protocol. 
A

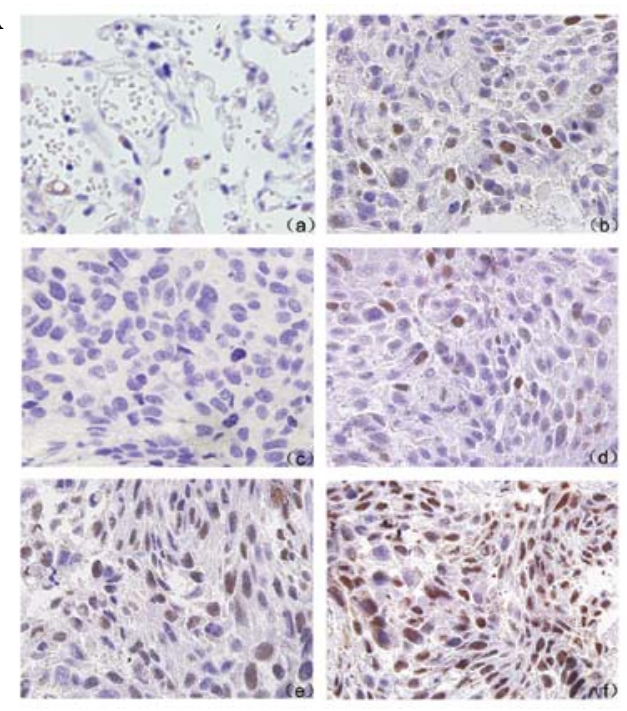

B
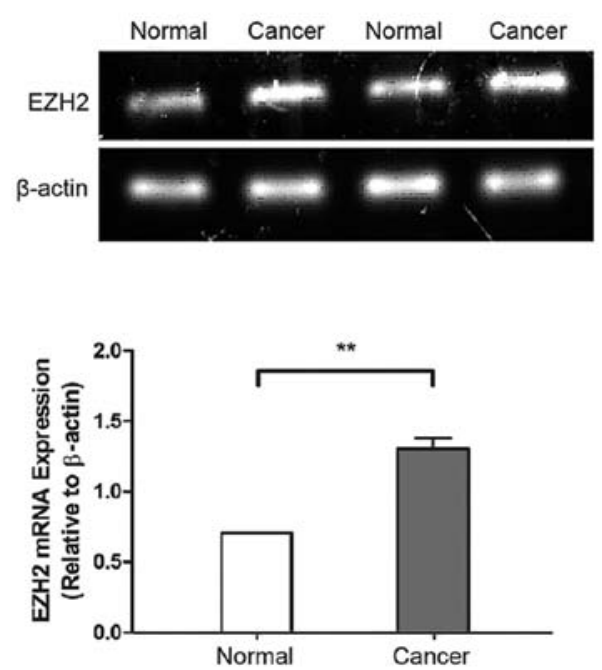

Figure 1. EZH2 expression in LA specimens and normal tissues were analyzed. (A) Immunohistochemical staining of EZH2 expression in normal and cancerous lung tissues. A representative is shown of normal, and cancer (negative and positive expression) tissues. a, normal tissue (negative expression); $b$, cancerous tissue (positive expression); c, pStage I tumor tissue (negative expression); d, pStage I tumor tissue (weak expression); e, pStage II tumor tissue (moderate expression); f, pStage III tumor tissue (strong expression). (B) RT-PCR analyses of EZH2 mRNA in normal and cancerous lung tissues. A representative result is shown. The relative quantitation of EZH 2 mRNA ${ }^{* * *} \mathrm{P}<0.001$ and ${ }^{* *} \mathrm{P}<0.01$ vs. normal tissues. Data are compiled from 3 independent experiments in each condition.

Table II. The expression of EZH2 in lung adenocarcinoma and distant normal tissues.

\begin{tabular}{lcccc}
\hline & & \multicolumn{2}{c}{ EZH2 expression } & \\
\cline { 3 - 4 } Variables & $\mathrm{n}$ & Positive & Negative & P-value \\
\hline Distant normal tissues & 69 & 8 & 61 & $<0.01$ \\
Lung adenocarcinoma & 69 & 44 & 25 & \\
\hline
\end{tabular}

The mRNA was reverse-transcribed to cDNA by using the PrimeScript RT-PCR kit (Takara). The primers used for the RT-PCR are shown in Table I. PCR products were separated on a $2 \%$ agarose gel and viewed by ethidium bromide (EB; Molecular Probes, Eugene, OR, USA) staining. The data were analyzed using AlphaImager 2200 software (Alpha Innotech, San Leandro, CA, USA).

Western blot analysis. Western blot analysis was performed as previously described (14). Cells were lysed with RIPA lysis buffer (Beyotime Biotechnology). For protein assay, BCA protein assay kit (Beyotime Biotechnology) was used. Protein samples were electrophoresed on 12\% SDS-PAGE and then electroblotted onto polyvinylidene fluoride (PVDF, Millipore) membranes. Immunoblotting was performed using the following antibodies: EZH2 antibody (ab3748) (1:1000, Abcam, USA) or monoclonal anti- $\beta$-actin antibody (1:4000, A1978, Sigma). Membranes were further incubated with polyclonal goat anti-mouse HRP-conjugated secondary antibody (Santa Cruz Biotechnology, Santa Cruz, CA, USA) for $2 \mathrm{~h}$ at room temperature. Bands were detected by an ECL kit (Cell Signaling). The intensity of each band was quantified using ImageJ 1.32 software (National Institutes of Health, Bethesda, MD, USA).
Flow cytometry. All fluorescence-conjugated antibodies used in this study were purchased from BD Bioscience. Trypsintreated cells were washed with PBS and fixed in 70\% ethanol overnight. Cells were then washed with PBS again and stained with propidium iodide (PI) $(50 \mu \mathrm{g} / \mathrm{ml})$ solution for cell cycle analysis. Cells were Annexin V-FITC and PI treated as recommended by the supplier. Cells were analyzed by flow cytometry using a flow cytometer (BD FACSCalibur, MI). To analyze intracellular proteins, cells were first fixed and permeabilized, and then stained with appropriate mAbs using a Cytofix/Cytoperm plus kit (eBioscience, San Diego, CA, USA). Flow cytometric analysis was performed using FlowJo software (Tree Star Inc., San Carlos, CA, USA).

Statistical analysis. Data are presented as the mean \pm SD, SPSS software package (versions 13.0, SPSS, Inc., Chicago, IL, USA) was used for all statistical analysis. Correlations of EZH2 expression with clinicopathological factors were examined by $\chi^{2}$ tests. Other data from experiments were analyzed by Student's t-test or ANOVA wherever appropriate. Difference was considered significant when the P-value was $<0.05$.

\section{Results}

EZH2 overexpression in LA tissues. In our study, EZH2 was detected as nuclear staining of the cells. EZH2 expression was detected in $44(63.8 \%)$ tumor tissues, including 12 cases (27.2\%) with weak expression (1+), 18 cases $(40.9 \%)$ with moderate expression (2+) and 14 cases $(31.8 \%)$ with strong expression (3+-4+). However, EZH2 expression was only detected in $8(11.6 \%)$ corresponding distant normal tissues (Fig. 1A; Table II).

In addition, we measured EZH2 mRNA expression with RT-PCR. It turned out to be consistent with those observed by immunohistochemistry (Fig. 1B). 
Table III. Relation between expression of EZH2 and clinicopathological characteristics of patients with surgically resected lung adenocarcinoma.

\begin{tabular}{|c|c|c|c|c|}
\hline \multirow[b]{2}{*}{ Variables } & \multirow[b]{2}{*}{ No. of cases } & \multicolumn{2}{|c|}{ EZH2 expression } & \multirow[b]{2}{*}{ P-value } \\
\hline & & Negative & Positive & \\
\hline Gender & & & & 0.601 \\
\hline Male & 36 & 12 & 24 & \\
\hline Female & 33 & 13 & 20 & \\
\hline Age (years) & & & & 0.532 \\
\hline$\leq 59$ & 27 & 11 & 16 & \\
\hline$>59$ & 42 & 14 & 28 & \\
\hline Tobacco history & & & & 0.037 \\
\hline Smoker & 39 & 10 & 29 & \\
\hline Never smoke & 30 & 15 & 15 & \\
\hline TNM stages & & & & 0.01 \\
\hline I & 22 & 15 & 7 & \\
\hline II & 26 & 5 & 21 & \\
\hline III & 21 & 6 & 15 & \\
\hline Pathology grade & & & & 0.036 \\
\hline Well & 22 & 12 & 10 & \\
\hline Moderate & 28 & 10 & 18 & \\
\hline Poor & 19 & 3 & 16 & \\
\hline
\end{tabular}

Moreover, the association of EZH2 expression with clinicopathological parameters was further analyzed. It showed that the EZH2 expression was significantly correlated with tobacco history $(\mathrm{P}=0.015)$, TNM stages $(\mathrm{P}=0.01)$, and pathology grade
$(\mathrm{P}=0.036)$ (Table III), which indicated that the EZH2 was involved in tumor development.

Elevation of EZH2 expression in drug-resistant LA cancer cells. To investigate the role of EZH2 in drug resistance, we measured EZH2 expression in a cisplatin-resistant LA cell line (A549/DDP) and a cisplatin-sensitive cell line (A549). Enhanced expression of EZH2 mRNA was observed in A549/ DDP cells compared with that in A549 cells (Fig. 2A). These findings were further confirmed by western blot analysis (Fig. 2B).

Suppression of EZH2 inhibited LA cell proliferation and migration. To understand the role of EZH2 in cell proliferation and migration, we knocked down EZH2 gene expression by shRNA interference. An obvious decrease of EZH2 mRNA level was observed in EZH2-knockdown cells (Fig. 3A).

Transfection of EZH2-targeting construct (shEZH2) into cells resulted in lower proliferation both in A549 and A549/ DDP compared with controls (Fig. 3B). Meanwhile, decreased migration was also observed both in A549 and A549/DDP cells after shEZH2 transfection (Fig. 4).

Suppression of EZH2 causes LA cell cycle arrest and apoptosis acceleration. Flow cytometry was used to assay cell cycle and apoptosis, we found that transfection of shEZH2 into A549 and A549/DDP cells led to G(2)/M cell cycle arrest (Fig. 5A) and increased apoptosis in EZH2-depleted cancer cells (Fig. 5B).

Suppression of EZH2 sensitized LA cells to cisplatin and decreased MRP1 expression. To investigate the effect of EZH2 depletion on cisplatin chemoresistance, MTT assays were performed. Cell survival was significantly inhibited after DDP treatment in A549/DDP-shEZH2 cells compared to control
$\mathbf{A}$
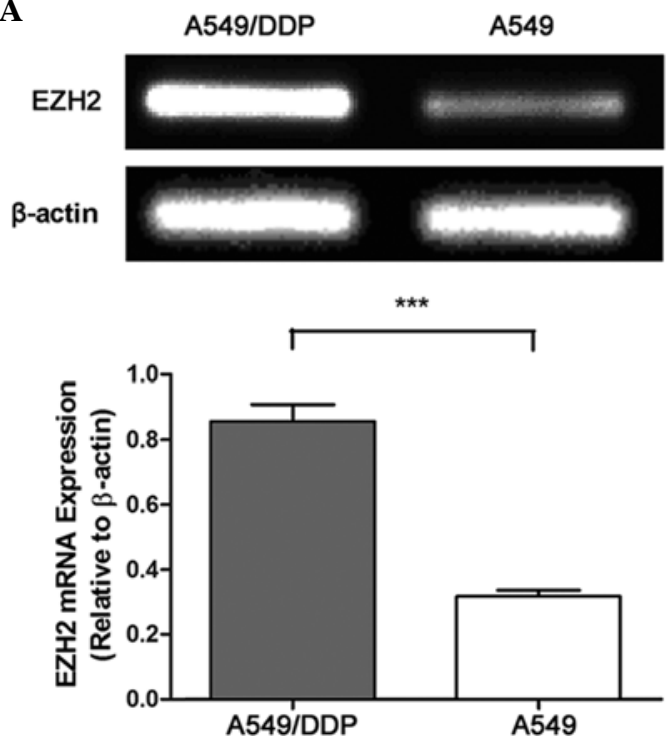

B
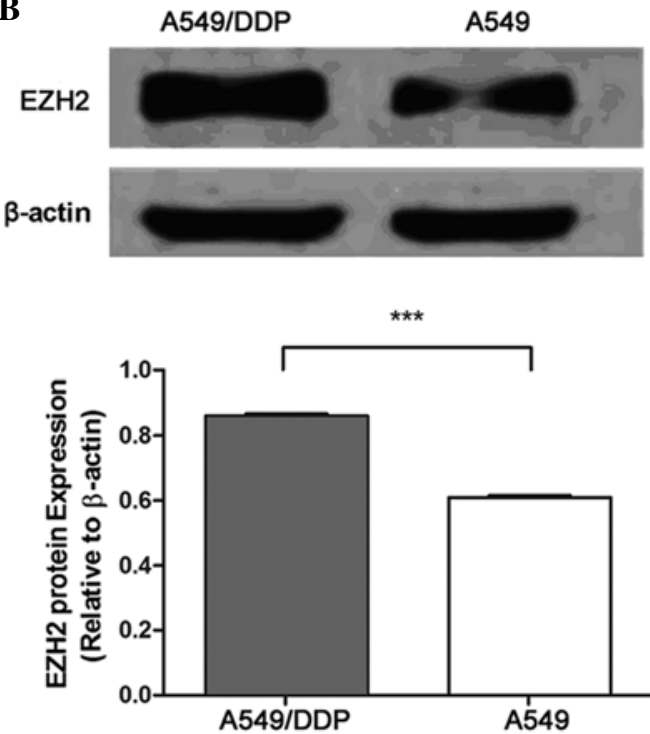

Figure 2. EZH2 expression in A549/DDP and A549 cell lines were analyzed. (A) The mRNA level of EZH2 in A549/DDP and A549 cell lines were analyzed. The relative level of EZH2 mRNA was upregulated in A549/DDP cells compared with A549 cells by RT-PCR. Data represent the mean and the standard deviation from three independent experiments. ${ }^{* * *} \mathrm{P}<0.001$ vs. A549 cells. (B) The protein level of EZH2 in A549/DDP and A549 cell lines were analyzed. The level of EZH2 protein was upregulated in A549/DDP cells compared with A549 cells by western blot analysis. Data represent the mean and the standard deviation from three independent experiments. ${ }^{* * *} \mathrm{P}<0.001$ vs. A549 cells. 
A
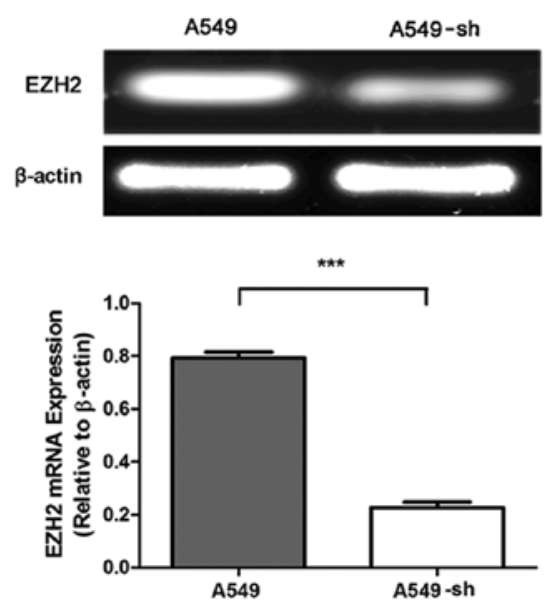

C

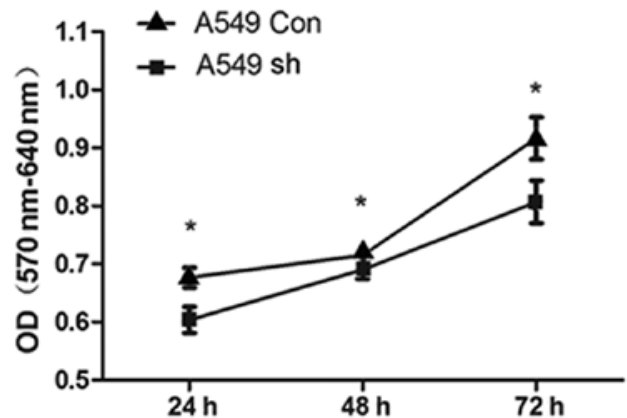

B
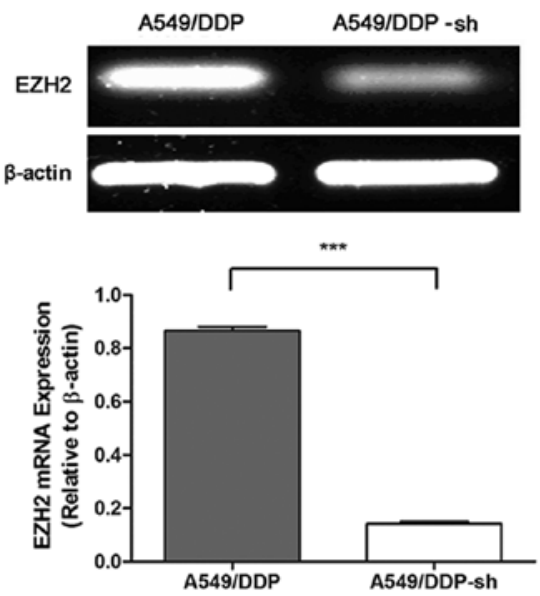

D

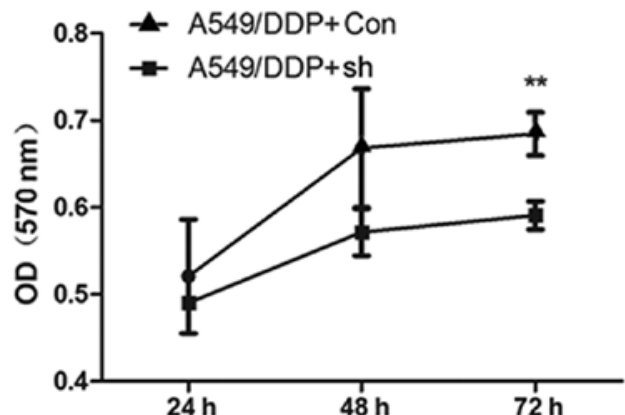

Figure 3. The stable transfection of EZH2-shRNA and the effect of EZH2 depletion on cell proliferation in A549 and A549/DDP cells were analyzed. (A) The EZH2-shRNA stable transfection in A549 cell line. Expression of EZH2 mRNA was decreased in shEZH2-A549 cells compared with control A549 cells by RT-PCR. Data represent the mean and the standard deviation from three independent experiments. ${ }^{* * *} \mathrm{P}<0.001$ vs. untransfected cells. (B) The EZH2-shRNA stable transfection in A549/DDP cell line. Expression of EZH2 mRNA was decreased in shEZH2-A549/DDP cells compared with control A549/DDP cells by RT-PCR. Data represent the mean and the standard deviation from three independent experiments. ${ }^{* * *} \mathrm{P}<0.001$ vs. untransfected cells. (C) The effect of EZH2 depletion on cell proliferation in A549 cells. EZH2 depletion in A549 cells significantly inhibited cell proliferation when compared with control cells. ${ }^{*} \mathrm{P}<0.05$ vs. control cells. (D) The effect of EZH2 depletion on cell proliferation in A549/DDP cells. EZH2 depletion in A549/DDP cells significantly inhibited cell proliferation on $72 \mathrm{~h}$ when compared with control cells. ${ }^{* *} \mathrm{P}<0.01$ vs. control cells.

A

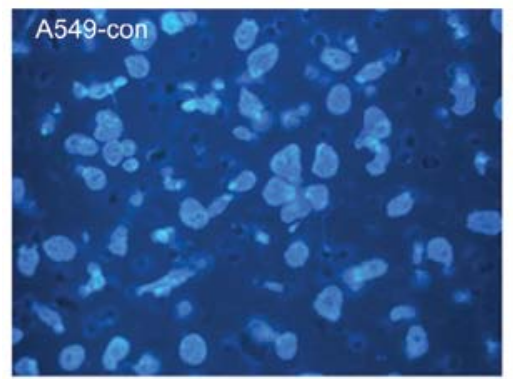

B

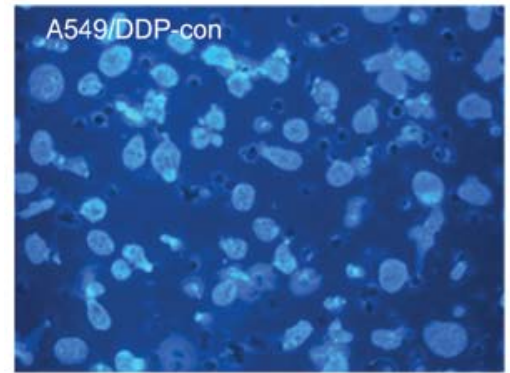

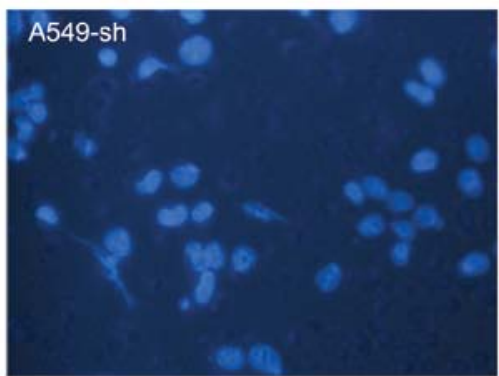
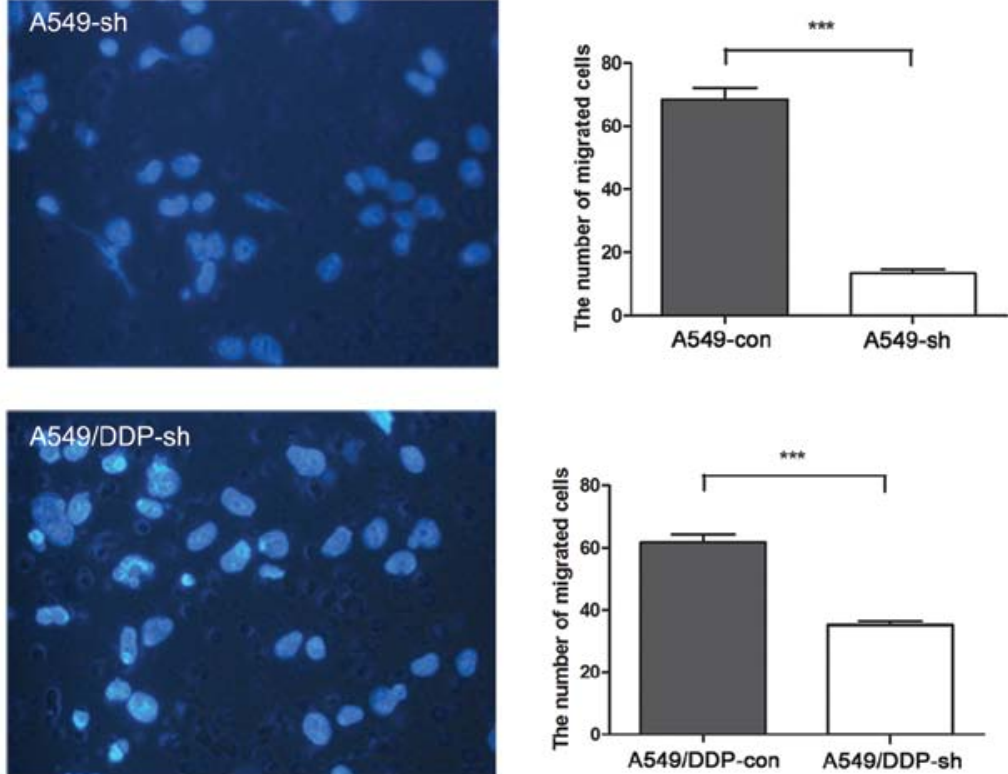

Figure 4. The effect of EZH2 depletion on cell migration in A549 and A549/DDP cells were analyzed. (A) The effect of EZH2 depletion on cell migration in A549 cells. Cells invading the polycarbonate membrance of transwell chamber was 87.5 \pm 3.3 and 23.5 \pm 5.1 per high power field in A549-con and A549-sh group, respectively $\left({ }^{* * *} \mathrm{P}<0.001\right)$. (B) The effect of EZH2 depletion on cell migration in A549/DDP cells. Cells invading the polycarbonate membrane of transwell chamber was $90.2 \pm 4.3$ and $25.1 \pm 4.5$ per high power field in A549/DDP-con and A549/DDP-sh group, respectively $\left({ }^{* * *} \mathrm{P}<0.001\right)$. 
A
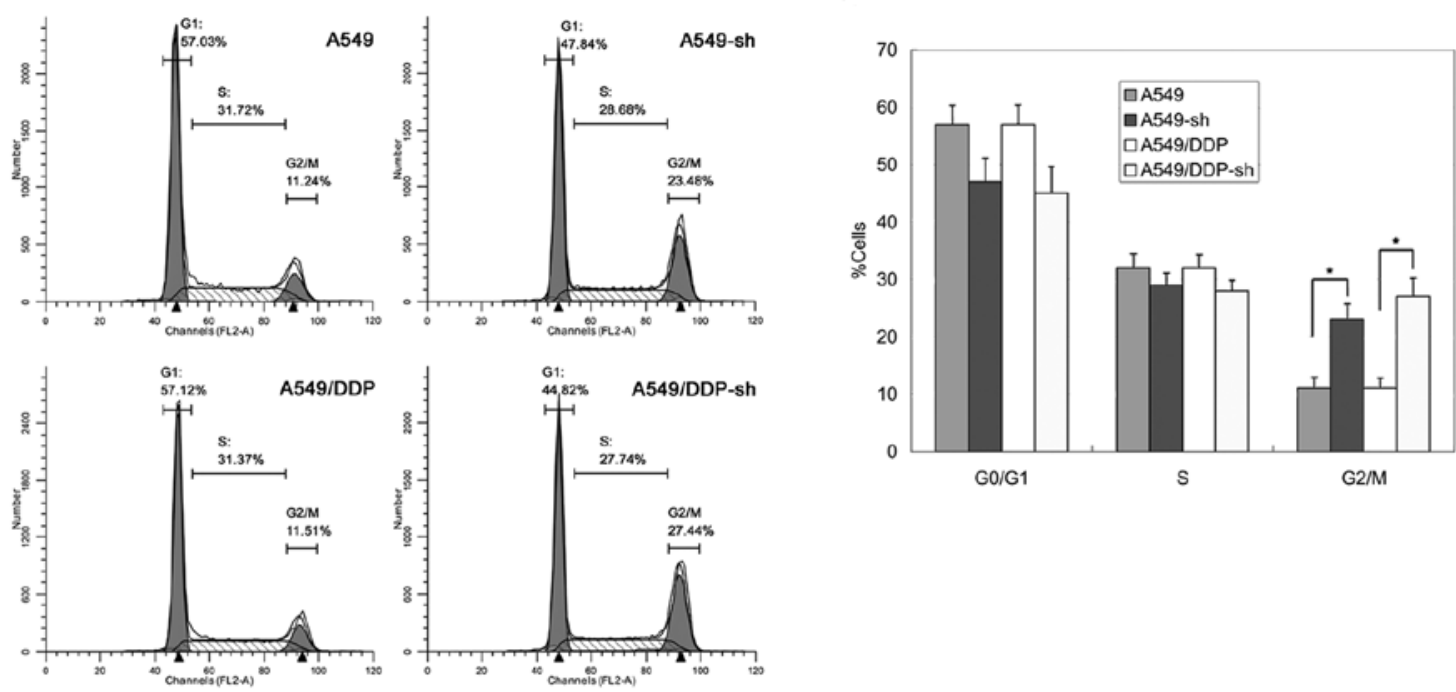
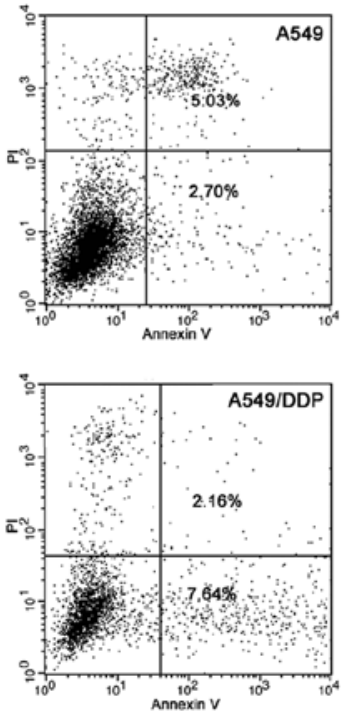
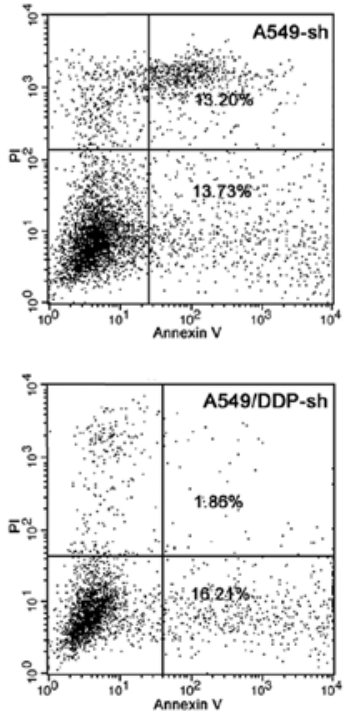

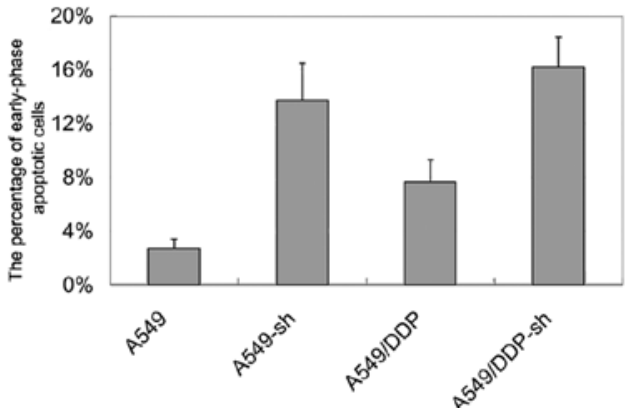

Figure 5. The effect of EZH2 depletion on cell cycle and apoptosis in A549 and A549/DDP cells were analyzed. (A) Effect of EZH2 depletion on cell cycle in A549 and A549/DDP cells. The DNA content was analysis by flow cytometry. Samples were fixed and stained with PI. Histograms show cell cycle distribution. (B) Effect of EZH2 depletion on cell apoptosis in A549 and A549/DDP cells. The percentage of apoptotic cells was determined by Annexin V-FITC/PI staining. Data were compiled from 3 independent experiments in each condition.

cells. The cisplatin $\mathrm{IC}_{50}$ was lower in A549/DDP-shEZH2 (90.400 $\mu \mathrm{M})$ cell vs. control cells $(131.2 \mu \mathrm{M})$. EZH2 deletion sensitized cancer cells to cisplatin, leading to a significant decrease of $\mathrm{IC}_{50}$ (Fig. 6A).

Furthermore, to study the possible mechanism of EZH2 in drug resistance, we measured mRNA level of MDR1, MRP1, and LRP expression. We found EZH2 deletion led to a down-regulation of MRP1 in A549/DDP-shEZH2 cells (Fig. 6B). EZH2 deletion did not have significant influence on the expression of MDR1 and LRP (data not shown).

\section{Discussion}

In the current study, we found that EZH2 expression was increased in tumor tissues compared to normal tissues. EZH2 expression was related to tumor pTNM stage. To investigate the potential role of EZH2 in LA, we knocked down EZH2 in LA cells with shRNA, and found that silencing of EZH2 gene inhibited cell proliferation, migration, arrested G2/M cell cycle and increased apoptosis. These results suggested that EZH2 may participate in tumor progression and influence cell function in LA cell lines. Moreover, we found that EZH2 was significantly expressed in the LA cell line resistant to cisplatin. EZH2 deletion sensitized cancer cells to cisplatin, leading to a significant decrease in survival of A549/DDP cells.

$\mathrm{PcG}$ genes (PcGs) play a key role in maintenance of cell identity. EZH2 gene, encoding a PcG protein, is also important in regulating cancer cell behaviour. The EZH2 protein is a member of the polycomb-complexes with histone lysine methyltransferase (HKMT) activity associated with transcriptional repression (15). Recent studies showed EZH2 was overexpressed in several human malignancies, and the level of EZH2 expression was correlated with cancer aggressiveness $(7,16,17)$. The correlation between EZH2 expression 
A

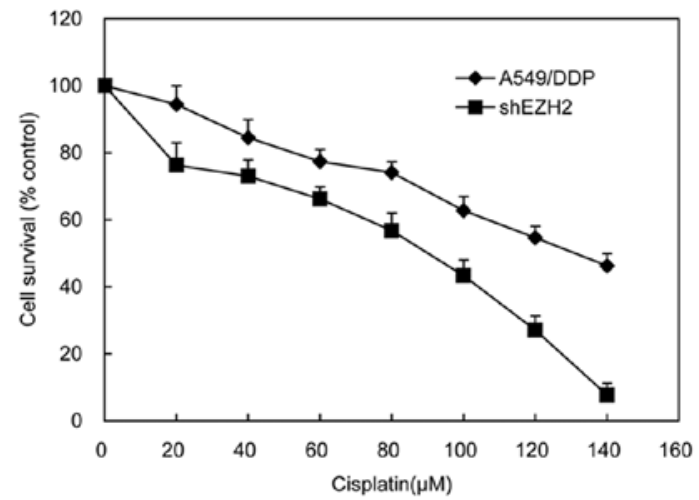

B
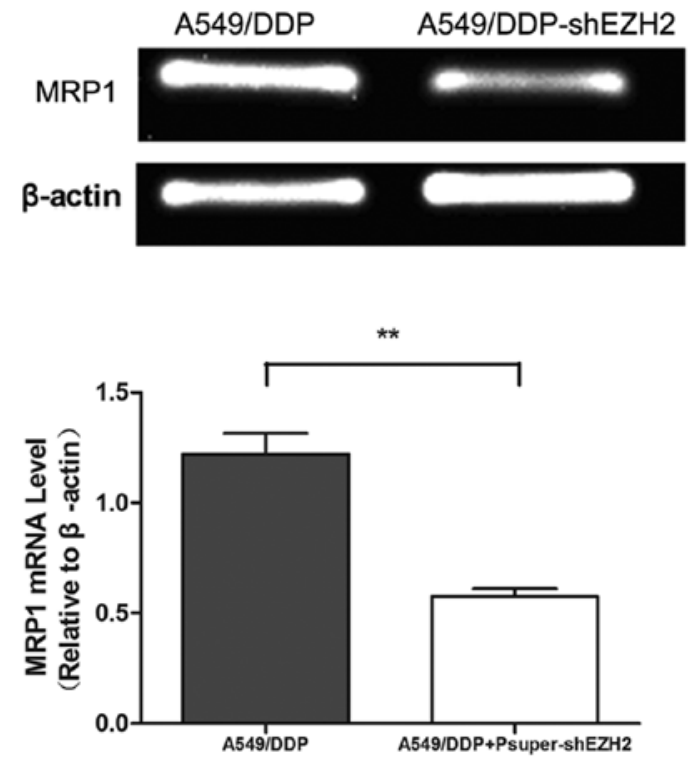

Figure 6. The effect of EZH2 depletion on DDP chemoresistance and mRNA expression of MRP1 in A549/DDP cells were analyzed. (A) The effect of EZH2 depletion on DDP chemoresistance. Cells were treated with various concentrations of DDP for $3 \mathrm{~h}$ followed by a 3-day recovery period. MTT assays were performed to assess chemoresistance. Cell survival was significantly inhibited after DDP treatment in A549/DDP-shEZH2 cells compared with control cells. The DDP IC $_{50}$ was significantly lower in A549/ DDP-shEZH2 $(90.400 \mu \mathrm{M})$ cells vs. control cells $(131.2 \mu \mathrm{M})$. (B) The effect of EZH2 depletion on mRNA level of MRP1. The relative level of MRP1 mRNA was downregulated in A549/DDP-shEZH2 cells compared with A549/DDP by RT-PCR. Data represent the mean and the standard deviation from three independent experiments. ${ }^{* *} \mathrm{P}<0.01$ vs. control cells.

and tumor aggressiveness/poor survival in those studies was consistent with our current observations in LA. Likewise, upregulated EZH2 expression was correlated with aggressive tumor behavior in patients with other malignant tumors such as prostate cancer (8), breast cancer (6) and gastric cancer (16). Our results extend the spectrum of tumors associated with EZH2. EZH2 expression is correlated with LA progression and may provide a novel way of diagnosis and prognosis of LA.

PcG proteins Bmi1 and EZH2 are epigenetic chromatin modifiers involved in cancer development (18), and the high EZH2 expression localized to primitive malignant cell types is often combined with a high Bmil expression $(19,20)$. EZH2 may mediate increased invasiveness and metastasis by silencing downstream target adrenergic receptor $\beta$-2 (ADRB2) (21). Recent studies indicated that EZH2 mediated transcriptional silencing of a tumor suppressor gene E-cadherin by trimethylation of $\mathrm{H} 3$ lysine 27 (22,23). How the EZH2 gene affects downstream genes that are involved in oncogenesis and progression need to be clarified.

Recently, Hussain et al reported that tobacco smoke engaged polycomb repressor complexes that contained EZH2 to mediate epigenetic silencing of Dickkopf-1 (a Wnt signaling antagonist) and enhanced the malignant phenotype of LC cells (24), which was in good agreement with our current observations that high expression of EZH2 was related with smoking.

Cisplatin, a commonly used therapeutic agent in NSCLC, together with a third-generation anticancer drug, such as vinorelbine, gemcitabine or taxanes, is the standard regimen used in the first-line treatment of advanced NSCLC. However, LA is less chemosensitive than other NSCLC. Our findings were consistent with another study showing that loss of EZH2-mediated methylation of histone H3 lysine 27 (H3K27) re-sensitized ovarian cancer cells to cisplatin (25). It was demonstrated that overexpression of EZH2 contributed to acquired cisplatin resistance in ovarian cancer cells in vitro and in vivo (26). Our results identified EZH2 as an important factor in LA cell chemoresistance for the first time. Abbosh et al (25) found that $\mathrm{H} 3 \mathrm{~K} 27$ methylation played an important role in drug resistance. EZH2 deletion sensitized pancreatic cancer cells to doxorubicin and gemcitabine (27), because EZH2 mediated H3K27 methylation is a mark of heterochromatin, and loss of chromatin compaction could allow increased DNA damage at lower doxorubicin and gemcitabine doses leading to a decreased survival in EZH2 deleted pancreatic cancer cells. Based on the above, we will focus on a possible change in $\mathrm{H} 3 \mathrm{~K} 27 \mathrm{me} 3$ levels with cisplatin resistance and how much it is reversed with EZH2 modulation in LA cells in our future studies.

We found that EZH2 downregulated the expression of MRP1 in LA cells. EZH2 deletion sensitized cancer cells to cisplatin, leading to a decrease of $\mathrm{IC}_{50}$. Our results suggested that overexpression of EZH2 might play a role in LA chemoresistance. The combination usage of EZH2 inhibitors could be superior to conventional therapy, and an approach to the enhancement of chemoresistance in LA.

In summary, our experiments indicated that EZH2 expression was upregulated in human LA tissues and functionally linked to the malignant behavior of LA cells. These results suggested that high EZH2 expression may be one of the candidates for new molecular diagnosis, prognosis and therapeutic targets in LA. Our study provided novel information on the mechanisms underlying the progression and chemoresistance of LA, and indicates a demand for development of pharmacological inhibitor of EZH2 as a potential anticancer agent for chemotherapy of LA.

\section{Acknowledgements}

Authors are grateful to Dr Lu-Tao Du, Cheng-Liu and XiaoJing Li for their technical guidance and data analysis (Qilu Hospital, Shandong University). The project was supported by the China National Natural Science Foundation Projects (no. 81001166), the Development of Science and Technology Plan Projects in Shandong Province (no. 2010GSF10283 and 2010GWZ2O242), and the Foundation of Shandong Public Health Department (no. 2009Q2012). 


\section{References}

1. Jemal A, Siegel R, Ward E, Hao Y, Xu J and Thun MJ: Cancer statistics, 2009. CA Cancer J Clin 59: 225-249, 2009.

2. Travis WD: Pathology of lung cancer. Clin Chest Med 32: 669-692, 2011

3. Montesinos J, Bare M, Dalmau E, et al: The changing pattern of non-small cell lung cancer between the 90 and 2000 decades. Open Respir Med J 5: 24-30, 2011.

4. Charloux A, Quoix E, Wolkove N, Small D, Pauli G and Kreisman $\mathrm{H}$ : The increasing incidence of lung adenocarcinoma: reality or artefact? A review of the epidemiology of lung adenocarcinoma. Int J Epidemiol 26: 14-23, 1997.

5. Parkin DM: Global cancer statistics in the year 2000. Lancet Oncol 2: 533-543, 2001.

6. Kleer CG, Cao Q, Varambally S, et al: $\mathrm{EZH} 2$ is a marker of aggressive breast cancer and promotes neoplastic transformation of breast epithelial cells. Proc Natl Acad Sci USA 100: 11606-11611, 2003.

7. Bachmann IM, Halvorsen OJ, Collett K, et al: EZH2 expression is associated with high proliferation rate and aggressive tumor subgroups in cutaneous melanoma and cancers of the endometrium, prostate, and breast. J Clin Oncol 24: 268-273, 2006.

8. Varambally S, Dhanasekaran SM, Zhou M, et al: The polycomb group protein $\mathrm{EZH} 2$ is involved in progression of prostate cancer. Nature 419: 624-629, 2002.

9. Rizzo S, Hersey JM, Mellor P, et al: Ovarian cancer stem cell-like side populations are enriched following chemotherapy and overexpress EZH2. Mol Cancer Ther 10: 325-335, 2011.

10. Berger W, Elbling L and Micksche M: Expression of the major vault protein LRP in human non-small cell lung cancer cells: activation by short-term exposure to antineoplastic drugs. Int J Cancer 88: 293-300, 2000.

11. Ikuta K, Takemura K, Sasaki K, et al: Expression of multidrug resistance proteins and accumulation of cisplatin in human non-small cell lung cancer cells. Biol Pharm Bull 28: 707-712, 2005.

12. Kourti M, Vavatsi N, Gombakis N, et al: Expression of multidrug resistance 1 (MDR1), multidrug resistance-related protein 1 (MRP1), lung resistance protein (LRP), and breast cancer resistance protein (BCRP) genes and clinical outcome in childhood acute lymphoblastic leukemia. Int J Hematol 86: 166-173, 2007.

13. Yuan C, Jiao L, Yang L, et al: The up-regulation of 14-3-3 proteins in Smad4 deficient epidermis and hair follicles at catagen. Proteomics 8: 2230-2243, 2008.

14. Liu H, Wang P, Song W and Sun X: Degradation of regulator of calcineurin 1 (RCAN1) is mediated by both chaperone-mediated autophagy and ubiquitin proteasome pathways. FASEB J 23: 3383-3392, 2009.
15. Kuzmichev A, Jenuwein T, Tempst $P$ and Reinberg D: Different EZH2-containing complexes target methylation of histone $\mathrm{H} 1$ or nucleosomal histone H3. Mol Cell 14: 183-193, 2004.

16. Matsukawa Y, Semba S, Kato H, Ito A, Yanagihara $\mathrm{K}$ and Yokozaki H: Expression of the enhancer of zeste homolog 2 is correlated with poor prognosis in human gastric cancer. Cancer Sci 97: 484-491, 2006.

17. Breuer RH, Snijders PJ, Smit EF, et al: Increased expression of the EZH2 polycomb group gene in BMI-1-positive neoplastic cells during bronchial carcinogenesis. Neoplasia 6: 736-743, 2004.

18. Valk-Lingbeek ME, Bruggeman SW and van Lohuizen M: Stem cells and cancer; the polycomb connection. Cell 118: 409-418, 2004.

19. Sasaki M, Ikeda H, Itatsu K, et al: The overexpression of polycomb group proteins Bmil and EZH2 is associated with the progression and aggressive biological behavior of hepatocellular carcinoma. Lab Invest 88: 873-882, 2008

20. Kikuchi J, Kinoshita I, Shimizu Y, et al: Distinctive expression of the polycomb group proteins Bmil polycomb ring finger oncogene and enhancer of zeste homolog 2 in nonsmall cell lung cancers and their clinical and clinicopathologic significance. Cancer 116: 3015-3024, 1915.

21. Yu J, Cao Q, Mehra R, et al: Integrative genomics analysis reveals silencing of beta-adrenergic signaling by polycomb in prostate cancer. Cancer Cell 12: 419-431, 2007.

22. Fujii S and Ochiai A: Enhancer of zeste homolog 2 downregulates E-cadherin by mediating histone $\mathrm{H} 3$ methylation in gastric cancer cells. Cancer Sci 99: 738-746, 2008.

23. Cao Q, Yu J, Dhanasekaran SM, et al: Repression of E-cadherin by the polycomb group protein EZH2 in cancer. Oncogene 27: 7274-7284, 2008.

24. Hussain M, Rao M, Humphries AE, et al: Tobacco smoke induces polycomb-mediated repression of Dickkopf-1 in lung cancer cells. Cancer Res 69: 3570-3578, 2009.

25. Abbosh PH, Montgomery JS, Starkey JA, et al: Dominantnegative histone $\mathrm{H} 3$ lysine 27 mutant derepresses silenced tumor suppressor genes and reverses the drug-resistant phenotype in cancer cells. Cancer Res 66: 5582-5591, 2006.

26. Hu S, Yu L, Li Z, et al: Overexpression of EZH2 contributes to acquired cisplatin resistance in ovarian cancer cells in vitro and in vivo. Cancer Biol Ther 10: 788-795, 2010.

27. Ougolkov AV, Bilim VN and Billadeau DD: Regulation of pancreatic tumor cell proliferation and chemoresistance by the histone methyltransferase enhancer of zeste homologue 2. Clin Cancer Res 14: 6790-6796, 2008. 